\title{
Multiresolution Plant Models with Complex Organs
}

\author{
Xiaopeng ZHANG* \\ LIAMA-NLPR \\ CAS Institute of Automation
}

Frédéric BLAISE ${ }^{\dagger}$

Marc JAEGER ${ }^{\ddagger}$

\author{
CIRAD sg-domf
}

LIAMA, CIRAD-AMAP

\begin{abstract}
Interactive visualization of virtual trees and forests is an important challenge in computer graphics and virtual reality. Complex geometric representation of virtual vegetation often overwhelms computer processing units and graphic hardware resources. We present here a new cluster based polygon decimation method, Hierarchical Union of Organs, to progressively and hierarchically simplify complex plant organs (foliage, flower, fruits). Using this method, we propose a novel approach to construct multiresolution organ geometrical models under spatial error control. During rendering, the approach generates minimal graphical primitives. The implementation shows its efficiency for geometrical compression on single trees. It also illustrates its fast rendering capabilities on scenes composed of numerous tree and plant species, including broadleaf and coniferous species.
\end{abstract}

CR Categories: I.3.5 [Computer Graphics]: Object hierarchies; I.3.7 [Computer Graphics]: Virtual reality

Keywords: polygon decimation, multiresolution, plant visualization, natural phenomena

\section{Introduction}

Social pressure on sustainable development has recently stimulated new multidisciplinary researches. Our concerns, which focus on modeling, simulation and visualization of the forest landscape, lie clearly within this frame work. With the development of novel techniques in plant modeling and simulation, many new plant modeling softwares have been produced. Thousands of plant species with fine geometric details can now be simulated. Fast visualization becomes more and more important as an assistant interface for agro-forestry management, virtual environment, ecosystem design, flight simulation, and digital recreation. Interactive and realistic visualization of plant communities has thus a challenging problem in computer graphics [Decaudin and Neyret 2004]. Trees and plants are complex objects in terms of shape, color and illumination, with many details of small, sparse and non-manifold geometry. The difficulties arise from the fact that the geometric shapes included in virtual natural scenes lead to a heavy burden of graphical primitives to handle.

Key points we want to address here are the rendering speed and memory costs of plant and tree organs (foliage, flower, fruits). Dedicated multiresolution methods were developed in recent years [Re-

\footnotetext{
*e-mail:xpzhang@nlpr.ia.ac.cn

†e-mail:frederic.blaise@cirad.fr

†e-mail:jaeger@liama.ia.ac.cn
}

molar et al. 2002], but had strong restrictions, such as leaf representation limited to a single quadrilateral and high pre-processing cost [Zhang and Blaise 2003]. In this paper we extend such approaches of foliage simplification to complex organ shapes, reducing pre-processing costs, and we apply the proposed approach to fast visualization of heterogeneous plant scenes.

\section{Related work}

Successful plant modeling systems have been developed in recent years, many of which are widely used.

AMAP models [de Reffye et al. 1988], include the botanical knowledge of plant architecture and real measured data, reflecting the plant's growing mechanism, including its spatial occupation, and the position of leaves, flowers or fruits. [Prusinkiewicz and Lindenmayer 1990] L-system is developed to describe the growth process of living organisms based on fractal patterns. L-system grammars are currently popular methods describing branching patterns and plant-growing processes. Xfrog [Lintermann and Deussen 1999] is a combination of a rule-based approach with traditional geometric modeling techniques. Plant models simulated in L-system and AMAP are representive of to real plants. Specific user guided LOD capabilities exist in those approaches, but none support automated and continuous multi-resolution models.

Polygon models are popular representations used for plant geometry. Some approaches have been developed to convert polygon models to other graphic primitives such as points and lines, impostors, and volumetric textures or volume spaces. Point and line models are efficient for rendering small polygons [Deussen et al. 2002] since the number of world-to-screen project transformations can be reduced. Impostors are used to convert complex geometries to simple ones with texture mapping. It is thus efficient for rendering repetitive objects in a scene [Shade et al. 1996]. Volumetric texture and volume methods are efficient for interactive realistic image synthesis of repetitive geometry [Decaudin and Neyret 2004]. However major drawbacks do arise from these techniques, in particular, close view are not possible and geometrical information can become lost.

Several methods have been proposed in the past to address polygon decimation, most of which are multiresolution modeling. We cite here only plant related works, since generic approaches based on object surface continuity fail. The Foliage Simplification Algorithm (FSA) [Remolar et al. 2002] aims at diminishing the number of leaf polygons in the crown, whilst maintaining their appearance, introducing the idea of leaf collapse. The new leaves obtained after collapsing preserve an area similar to the original ones. Progressive Leaves Union (PLU) [Zhang and Blaise 2003] is a modified FSA to keep more reasonable spatial occupation and color distribution of the foliage with measurements of similarities. A common drawback of FSA and PLU is that only quadrilateral leaves are processed. More complex leaves, flowers, and fruits cannot yet be modeled.

In this paper, we present an upgrade and modification of $F S A$ and $P L U$. Section 3 shows how these concepts are used to define hierarchical union of organs. Section 4 is dedicated to error controlled 
multiresolution plant geometry, while section 5 illustrates results.

\section{Hierarchical Union of Organs}

We aim here to define a process able to generate multi-resolution representations of tree organs with respect to botanical concepts. Structure, position and orientation in organs, related to botanical knowledge, have a major effect on organ appearance.

\subsection{Principles}

From botanical knowledge [de Reffye et al. 1988] we know that phyllotaxy and anthotaxy provide basic information about leaves and flower structures. We want to simplify foliage, or more generally organs, in respect of the pre-cited botanical concepts: leaf, petal phyllotaxy, flower anthotaxy, leaf and petal structure and distribution. The proposed new method should have structural consistency, inducing local clustering inside a phyllotaxy group, an anthotaxy group, or a flower group.

The proposed algorithm, Hierarchical Union of Organs (HUO) generates different degrees of simplified geometric shapes of the original plant model for its multiresolution representation. It includes four steps. The first step is to organize the plant organs into groups corresponding to leaf phyllotaxy, flower anthotaxy and the distribution of petals in a flower. The second is to decompose complex mesh models of plant organs into independent quadrilaterals and triangles, building organ clusters. The third step is a recursive union process to construct new quadrilaterals inside each cluster, until reaching a single quadrilateral, the representative quadrilateral of this cluster. The fourth step performs foliage union on all representative quadrilaterals.

With this algorithm, all shapes of plant organs can be simplified, and when coupling it with multiresolution branch representation, it leads to an efficient multiresolution plant geometry description.

\subsection{Progressive union of complex organs}

The idea of leaf collapse in FSA [Remolar et al. 2002] and $P L U$ [Zhang and Blaise 2003] are inherited here and extended to include triangles, named as Progressive Union of Complex Organs. Each step of this progressive union constructs a new quadrilateral from a pair of quadrilaterals or triangles, i.e., choosing four vertices from the vertex list $L$ of six, seven or eight vertices that are the vertices of the polygon pair.

The rule for simplification can be expressed as follows: (1) Find two vertices from $L$ of longest distances, giving the two vertices of the main axis of the new leaf; (2) Find a vertex, noted as point $C$, from the remaining list $L$ having the biggest sum of distances to $A$ and $B ;(3)$ Find a vertex, noted as point $D$, from the remaining list $L$ having the biggest sum of distances to $A, B$, and $C$; (4) Translate $C$ and $D$ so that their center coincides with center of segment $A B$, constraining the new quadrilateral to be planar; (5) Keep the original normal vectors at points $A$ and $B$, and compute normals at points $C$ and $D$ from their positions.

Therefore, the final quadrilateral is a parallelogram, keeping the local maximum expansion of all the decimated organs, and guaranteeing low distortion on the new leaf. Figure 1 shows the process of polygon union and polygon separation. The resulting quadrilateral

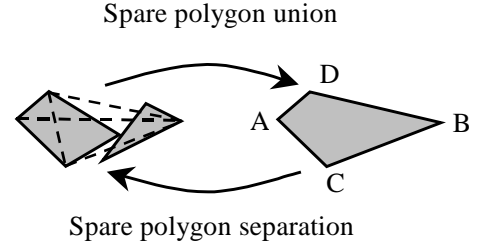

Figure 1: Progressive union of two polygons

is stored with reference to the original quadrilaterals or triangles in order to allow reverse Polygon Separation on rendering request.

This is a recursive process of destroying old leaves and generating new ones until a single leaf is left. Since all the leaves are useful in representing the plant crown at different resolutions, all these leaves are recorded as the result of pre-processing.

\subsection{Hierarchical union among sparse clusters}

We apply now the Progressive Union Complex Organs process recursively at different levels of the plant, building an Hierarchical Union of Organs(HUO). The first level is confined to each single organ, so that all primitives representing the geometry of this organ cluster are simplified and the general visual effect of this organ is kept. The second level is confined inside each phyllotaxy and anthotaxy group. The third level is recursively applied in each tree or plant until reaching a single quadrilateral. The different classes of organ (leaf, flower, and fruit) are processed separately since their material properties are usually different. Figure 2 illustrates the progressive union in a phyllotaxy group.

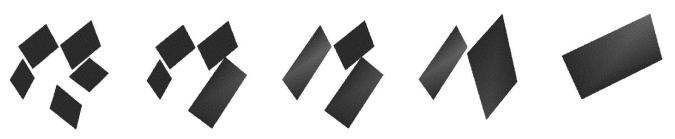

Figure 2: Progressive union in a phyllotaxy group

The error for each union is useful for the construction of multiresolution models. Equation (1) defines the error for each union of the distances from old vertices to its projection on the new polygon:

$$
d\left(P_{i}, \pi\right)=\left\|\operatorname{Proj}_{\pi}\left(P_{i}\right)-P_{i}\right\|
$$

where $\operatorname{Proj}_{\pi}$ stands for the projection made on the plane enclosing the polygon $\pi$. If the projection point is not inside the polygon, projection is made on its edges.

We detail hereby how we establish the cost of the polygon union to chose the best pair to decimate. The calculation of the cost for polygon union of polygon $\pi_{1}$ and polygon $\pi_{2}$ is given by (2).

$$
C\left(\pi_{1}, \pi_{2}\right)=\sum_{i=1}^{6} k_{i} \times S_{i}\left(\pi_{1}, \pi_{2}\right)
$$

where $S_{1}$ is normal similarity, $S_{2}$ is positional similarity, $S_{3}$ is area similarity, $S_{4}$ is diameter similarity, $S_{5}$ is union age similarity, $S_{6}$ is diameter penalty, and $k_{i}$ are weights, as defined in [Zhang and Blaise 2003], since its effect is satisfactory in terms of visual effect.

In the end of pre-processing, the different organ geometrical levels and their structure are stored on the disk for further use in rendering. 


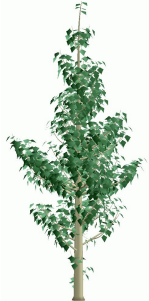

(a) 7520

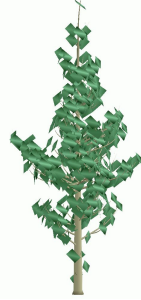

(b) 1504

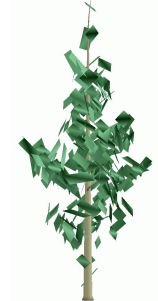

(c) 804

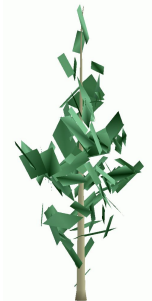

(d) 400
Figure 3: Hierarchical foliage union

Each record contains classical vertices, normals, polygon lists and the union process list with its corresponding error.

Figure 3 shows the effect of hierarchical union with its corresponding polygon number. Figure 3(a) is the original model, Figure 3(b) is the result of the first of $H U O$, Figure 3(c) and Figure 3(d) are some intermediate results of the third level of $H U O$.

\section{Error controlled graphical primitives}

On a given final image output, the requested pixel error can be converted to a spatial error, noted $\varepsilon$, defined from the viewing parameters. In our scene rendering implementation, the spatial error of each plant is calculated through the principle of central projection. From the preprocessed multiresolution models, lists of vertices, normal vectors, polygons, and union process can now be used for visualization under this spatial error control.

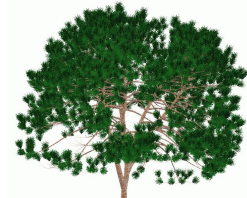

(a) 383800

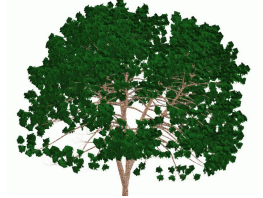

(b) 32904

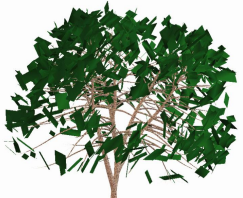

(c) 1484
Figure 4: Multiresolution Scots Pine with a complex crown

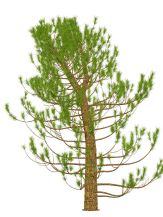

(a) 31920

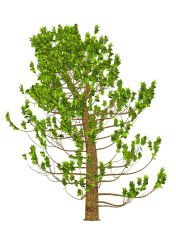

(e) 760

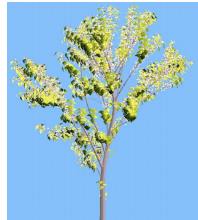

(b) 30212

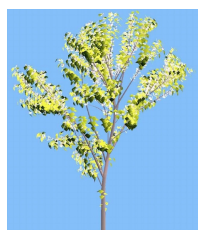

(f) 3236

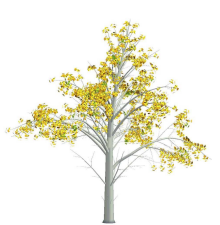

(c) 72490

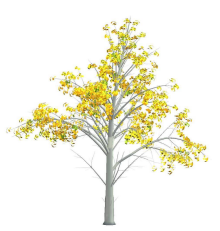

(g) 14498

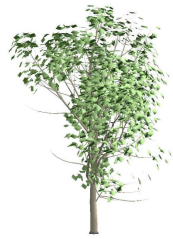

(d) 61056

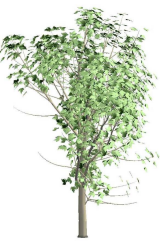

(h) 5512
Figure 5: Polygon number on original and simplified trees
Organs and branches are processed separately since their topology is quite different. Multiresolution geometry of branches is not detailed here, however, main ideas [Zhang and Jaeger 2006] are summarized as follows: branch geometry is described by a multiresolution model from equations of straight cylinder pieces, noted as internodes. According to the spatial error $\varepsilon$, internode surfaces are discretized and merged along the branch axis during rendering.

Concerning the other organs, based on the spatial error $\varepsilon$, the geometrical models of organs are also constructed on the fly, traversing the preprocessed multiresolution geometrical data set. Traversing stops when recorded error is smaller than $\varepsilon$ and corresponding graphical primitives are generated and sent to the GPU.

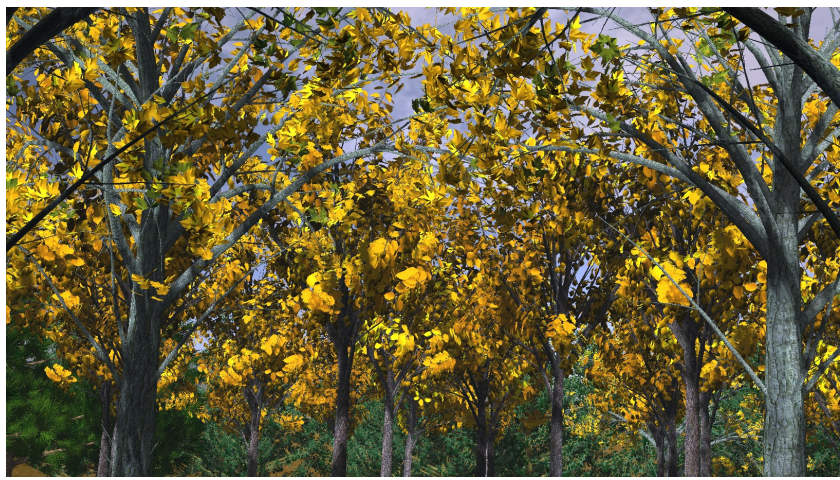

Figure 6: Visualization of a virtual garden

\section{Implementation and results}

All virtual trees used in this paper were generated using AMAP Genesis ${ }^{T M}$ Software. The implemented system is divided into two stages, both written in $\mathrm{C}$ language.

The preprocessing stage can be expressed as five different tasks:

Task 1 Data preparation: organ class definition (leaves, flowers, or fruits), with clustering tables corresponding to phyllotaxy, anthotaxy, or flower patterns groups;

Task 2 Hierarchical union of complex polygons to triangles and quadrilaterals in each organ with cost and error evaluation;

Task 3 Hierarchical union on botanical clusters, cost and error calculation;

Task 4 Hierarchical union on full tree until reaching a single polygon, including cost and error calculation;

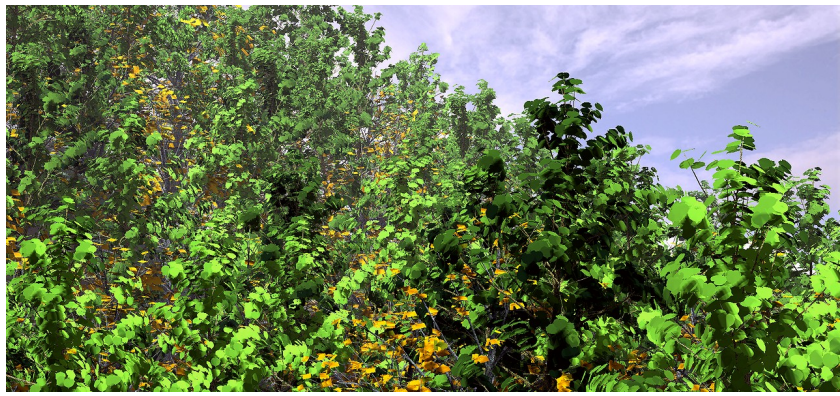

Figure 7: Multiresolution foliage on maple, poplar forest 
Task 5 Record of geometry, error, and union process on disk.

The single plant or scene retrieval stage outputs interactive 3D views, using OpenGL library. This application reads scene description, sorts plants according to their species, and, according to viewer position and user error control, generates and sends appropriate graphical primitives to the GPU from the pre-processed data. Fully clipped trees are ignored by simple bounding box test.

Images in this paper were all generated using a PC with a Pentium IV (E) PC 3.0G(HT)/Intel, memory 1GB, and video card NVIDIA GeForce FX 5700LE. Images were synthesized in nearly 10 frame per sec to 0.5 frame per sec for Figures 6 and 7. In these two figures, shadow was added by a Z-buffer post process. The weights $k_{i}$ of formula (2) are set as follow: $k_{1}=0.037, k_{2}=0.312, k_{3}=$ $0.037, k_{4}=0.037, k_{5}=0.287, k_{6}=0.287$.

We have tested our approach with error control algorithms on different tree species with needle-like leaves, broad leaves or complex shapes, thin leaves with complex shapes, flowers with complex shapes, and fruits. Figure 4 is an example of a multiresolution 25 year old Scots Pine $9 \mathrm{~m}$ high, $75 \mathrm{~m}$ away from the viewer, showing both tree aspect and corresponding number of folliage polygons from the original full detailed one in Figure 4(a) to the simplified one Figure 4(c). Other models are shown with polygon number in Figure 5 on a 45 year old Aleppo pine, a 12 year old white poplar, a 20 year old crab apple in the spring, and a 15 year old silver maple. The images in Figure 5(a), 5(b), 5(c), 5(d) are the original models, and the images in Figure 5(e), 5(f), 5(g), 5(h) are simplified ones. Compression ratios are $42.00,5.00,9.34$, and 11.08 respectively.

We apply our approach to virtual garden and forest scenes. Figure 6 is an example of an immerged view inside a virtual garden built from 83 trees with poplar trees, pine trees, apple trees and holly trees, the number of polygons of the original model is 9.5 million, and those of this multiresolution model is 1.2 million, so the compression ratio is 7.85 . Figure 7 is another example, showing a wild poplar and maple forest of 213 trees, on a sloping land. The landscape is designed through a random distribution of plants in arable areas. The number of polygons of the original model is over 42 million, the resulting multiresolution model delivers 317894 primitives, leading to a compression ratio of 134 . Related polygon numbers of clipped trees are not considered in these figures.

\section{Conclusions and further work}

We propose hereby a novel hierarchical plant organ multiresolution geometrical model. More precisely:

1. The proposed approach extends foliage simplification algorithms to complex shapes describing leaves, flowers, fruits;

2. Plant knowledge of organ phyllotaxy and anthotaxy are used to define plant organ clusters, hierarchically, in respect of object nature and geometry. The hierarchical structure and simplified representations of these clusters are therefore constrained by the original plant structure and organ nature. The visual effect of simplified plant models is thus kept.

3. The pre-processing time in organ geometrical union is drastically dropped, since simplification operations are held locally, at a given cluster hierarchical stage.

4. The approach allows multiresolution geometrical representation of trees with very significant compression ratios, up to a single leaf for a whole crown.
5. Implementation of the approach generates geometry at different LOD levels on the fly during rendering, under view and user control. Fast rendered virtual trees and virtual garden scenes were generated, showing the potential of the approach

The implementation of the proposed approach is a prototype. Up to now, texture is mapped only on branches. We introduce it on organs, trying to keep structural consistency and LOD properties, for further realism. The union algorithm is developed for leaves/petals of similar size in a given crown; it may be easily adapted for various scales depending on plant species. The approach could also be combined with progressive surface simplification. Specific models may be needed for needles in coniferous species or flower and fruiting components.

Moreover, since our approach is not dedicated to a specific plant generator, nor a specific rendering technique, various improvements aimed at real-time applications could be made e.g. point splat may be used from LOD clusters, or impostors at various cluster levels.

\section{Acknowledgements}

Thanks to Dr. Alexia Stokes for her overall check on English in this paper. The first two authors began this work at LORIA - INRIA Lorraine. This work is supported by National Natural Science Foundation of China projects No. 60073007, 60473110, 30371157; by the French National Research Agency within project NATSIM ANR- 05-MMSA-45, and by LIAMA funding with the project GreenLab.

\section{References}

De Reffye, P., Edelin, C., Françon, J., Jaeger, M., AND PUECH, C. 1988. Plant models faithful to botanical structure and development. In SIGGRAPH '88, vol. 22, 151-158.

Decaudin, P., AND NeYRet, F. 2004. Rendering forest scenes in real-time. In Rendering Techniques (Eurographics Symposium on Rendering), 93-102.

Deussen, O., Colditz, C., Stamminger, M., and DretTAKIS, G. 2002. Interactive visualization of complex plant ecosystems. In Proc. Conf. Visualization 2002, 219-226.

LINTERMANN, B., AND DEUSSEN, O. 1999. Interactive modeling of plants. IEEE Comput. Graph. Appl. 19, 1, 56-65.

Prusinkiewicz, P., AND Lindenmayer, A. 1990. The algorithmic beauty of plants. Springer-Verlag New York, Inc., New York, NY, USA.

Remolar, I., Chover, M., Belmonte, O., Rebollo, C., AND RibELles, J. 2002. Geometric simplification of foliage. In Eurographics Short Presentations, 397-404.

Shade, J., Lischinski, D., SAlesin, D. H., DeRose, T., AND SNYDER, J. 1996. Hierarchical image caching for accelerated walkthroughs of complex environments. In SIGGRAPH '96, 7582.

Zhang, X., And Blaise, F. 2003. Progressive polygon foliage simplification. In Proc. Plant Growth Modeling and Applications, Tsinghua University and Springer Press, 217-230.

ZHANG, X., AND JAEGER, M. 2006. Multi resolution tree branch modelling and rendering. Submitted to Eurographics 2006. 